\section{A BASIC program to randomly generate members of a weighted set}

\section{S. A. ROBERTSON \\ Manchester Polytechnic, Manchester, England M15 5FG}

Writing a program to randomly select and printout members of a given set (of letters, integers, words, etc.) is a relatively simple matter. All that is needed is an array in which the members may be stored, together with a random number generator which can generate numbers in the range 1 to $\mathrm{L}$, where there are $\mathrm{L}$ members in the set. If the members of the set are stored, for example, in Array Locations $\operatorname{Af(1)}$ to $\operatorname{Af(L)}$, we might have:

\section{$50 \mathrm{R}=\mathrm{INT}\left(\mathrm{L}^{*} \mathrm{RND}(0)\right)+1$ \\ 60 PRINT Af(R)}

as a simple solution to the problem of randomization which can be repeated for further selections.

If we wish to assign weights to the different members in the set, the problem becomes slightly more complex. We might wish, for instance, to set up a "letter generator" where the likelihood of each letter being selected is based upon its frequency. Similarly, we might wish to devise a "word generator" where the likelihoods of the various words in the set are to be determined by word frequency counts. The program presented provides a solution to this kind of problem.

Suppose we had the problem of randomly generating members of the set $A, B, C, D$, and $E$, where the weights to be applied were, respectively, 6, 7, 2, 5, and 13. The set itself can be stored in an Array $X \mathfrak{X}(\mathrm{I})$ (Locations 0 to 4 ), while the weights can be stored in an Array X(I) (again in Locations 0 to 4). The aim of the program is to assign a likelihood to each letter on the basis of its weighting, and this effectively means to allow the probability of any letter to equal $p_{i}$ such that:

$$
p_{i}=w_{i} / \sum_{i=1}^{L} w_{i}
$$

that is to say, the ratio of the size of its weight to the sum of the weights for all members in the set. The program achieves this in a relatively economical manner.

A BASIC Program. The routine first calculates the sum of all weights to give a total value $T$ (in our example, 33). Next, a random number ( $R$ ) is generated in the range 1 to $T$ and the weights for the individual members in the set are subtracted from this in a stepwise manner, starting with the first $X(0)$. The first weight to cause a zero or negative result in this subtraction identifies the set member to be generated. Hence, if $R$ was equal to 14 , stepwise subtraction of the weights
Table 1

Program to Randomly Generate Members of a Weighted Set

\begin{tabular}{|c|c|}
\hline 10 & REM WEIGHTED RANDOMIZATION PROGRAM \\
\hline 20 & DIM Xf(100),X(100) \\
\hline 30 & INPUT L,N \\
\hline 40 & FOR $I=0$ TO $L-1$ \\
\hline 50 & INPUT X£(I), X(I) \\
\hline 60 & IF $X(I)<=0$ THEN 280 \\
\hline 70 & NEXT I \\
\hline 80 & FOR $\mathrm{J}=0$ TO $\mathrm{L}-1$ \\
\hline 90 & IF INT $(X(J))=X(J)$ THEN 140 \\
\hline 100 & FOR $\mathrm{K}=0$ TO $\mathrm{L}-1$ \\
\hline 110 & LET $X(K)=X(K) * 10$ \\
\hline 120 & NEXT $K$ \\
\hline 130 & GOTO 90 \\
\hline 140 & NEXT J \\
\hline 150 & LET T $=0$ \\
\hline 160 & FOR $M=0$ TO $L-1$ \\
\hline 170 & LET $T=T+X(M)$ \\
\hline 180 & NEXT M \\
\hline 190 & FOR O $=1$ TO $\mathrm{N}$ \\
\hline 200 & LET R=INT $\left(T^{*}\right.$ RND $\left.(0)\right)+1$ \\
\hline 210 & LET $A=0$ \\
\hline 220 & LET $R=R-X(A)$ \\
\hline 230 & IF $R<=0$ THEN 260 \\
\hline 240 & LET $A=A+1$ \\
\hline 250 & GOTO 220 \\
\hline 260 & PRINT Xf(A) \\
\hline 270 & NEXT O \\
\hline 28 & STOP \\
\hline & END \\
\hline
\end{tabular}

from the first member onward would yield the results 8 and 1 before the third weight produced the solution -1 , thus identifying " $C$ " for generation. Had $R$ been 32 , the results of such subtraction would have been $26,19,17,12$, and -1 , leading to generation of $\mathrm{X}(4)$ or "E." Similarly, had R been $4, X f(0)$ or " $A$ " would have been the first member generated. The program repeats this procedure in making further selections.

The reasoning behind the routine is relatively straightforward. Each weight can be thought of as an interval in the range 1 to T. All numbers in this range are equally likely to be generated by the random number generator (with a probability of $1 / \mathrm{T}$ ). The likelihood of a number falling within any particular interval, however, will be directly dependent on the size of that interval $\left(w_{i}\right)$ relative to the total range $\left(T\right.$ or $\left.\underset{i}{L} w_{i}\right)$. The process of subtracting out weights from $R$ is merely a device for identifying the particular interval in which the random number has fallen.

The program makes use of integer values in its operation although real and/or integer values greater than zero may be input as the routine itself transforms the weights into an appropriate form. This is performed by repeated multiplication of the weights by 10 until they all have integer status. This procedure does not affect the relative probabilities involved. 
Since real values can be input, raw probabilities may be used as weights where it will be important to insure that $\sum_{i=1}^{L} w_{i}$ is equal to 1 or some positive power of 10 .

Program Input. The program first requires (Line 30 ) the number of members in the set or its length (L), together with the number of selections or generations required in the run $(\mathrm{N})$. After this, each member in the set and its weight can be input in a stepwise manner.

Computer. The program was developed on an
ICL $1902 S$ computer using a standard BASIC compiler. Availability. A listing of the program, together with a version dedicated to the generation of letter strings and associated output, may be obtained by contacting the author at the School of Psychology, Manchester Polytechnic, Gaythorn Annexe, River Street, Manchester, England M15 5FG.

(Accepted for publication July 5, 1977.) 